\title{
Pharmacokinetic Parameter Group Identifier
}

National Cancer Institute

\section{Source}

National Cancer Institute. Pharmacokinetic Parameter Group Identifier. NCI Thesaurus.

Code C87980.

A character or string that represents a pharmacokinetic parameter group. 